\title{
Pregabalin in the Management of Painful Diabetic Neuropathy: A Narrative Review
}

\author{
Shazli Azmi · Kariem T. ElHadd • Andrew Nelson · Adam Chapman • \\ Frank L. Bowling · Anughara Perumbalath · Jonathan Lim • \\ Andrew Marshall · Rayaz A. Malik · Uazman Alam
}

Received: September 28, 2018 / Published online: December 18, 2018

(C) The Author(s) 2018

\section{ABSTRACT}

Pregabalin is a first-line treatment in all major international guidelines on the management of painful diabetic neuropathy (pDPN). Treatment with pregabalin leads to a clinically meaningful improvement in pain scores, offers consistent relief of pain and has an acceptable tolerance level. Despite its efficacy in relieving neuropathic pain, more robust methods and comprehensive studies are required to evaluate its effects in relation to co-morbid anxiety and sleep interference in pDPN. The sustained

Enhanced Digital Features To view enhanced digital features for this article go to: https://doi.org/10.6084/ m9.figshare.7398635.

S. Azmi · F. L. Bowling - A. Marshall · R. A. Malik Institute of Cardiovascular Science, University of Manchester and Manchester Diabetes Centre, Manchester Foundation Trust, Manchester, UK

K. T. ElHadd

The Walton Centre, Liverpool, UK

A. Nelson - A. Chapman - A. Perumbalath · J. Lim . U. Alam ( $\square)$

Diabetes and Endocrinology Research, Department of Eye and Vision Sciences and Pain Research Institute, Institute of Ageing and Chronic Disease, University of Liverpool and Aintree University Hospital NHS Foundation Trust, Liverpool, UK e-mail: uazman.alam@liverpool.ac.uk;

Uazman.alam@manchester.ac.uk benefits of modulating pain have prompted further exploration of other potential target sites and the development of alternative GABAergic agents such as mirogabalin. This review evaluates the role of pregabalin in the management of pDPN as well as its potential adverse effects, such as somnolence and dizziness, which can lead to withdrawal in $\sim 30 \%$ of long-term use. Recent concern about misuse and an increase in deaths linked to its use has led to demands for reclassification of pregabalin as a class $\mathrm{C}$ controlled substance in the UK. We believe these demands need to be tempered in relation to the difficulties it would create for repeat prescriptions for the many millions of patients with pDPN for whom pregabalin provides benefit.

\section{U. Alam}

Division of Endocrinology, Diabetes and

Gastroenterology, University of Manchester,

Manchester, UK

R. A. Malik

Weill Cornell Medicine-Qatar, Doha, Qatar

U. Alam

Department of Diabetes and Endocrinology, Royal Liverpool and Broadgreen University NHS Hospital Trust, Liverpool, UK 
Plain Language Summary: Plain language summary available for this article.

Keywords: Anxiety; GABAergic therapy; Neuropathic pain; Painful diabetic neuropathy; Pregabalin; Sleep interference

\section{PLAIN LANGUAGE SUMMARY}

Diabetic peripheral neuropathy (DPN) is a disease of the nerves in the hands and feet and is a common complication of both type 1 and type 2 diabetes. The symptoms of DPN include loss of sensation, weakness and pain. Around $30-40 \%$ of people with diabetes have DPN, and its burden will rise with the exponential increase of diabetes worldwide. pDPN is a debilitating complication not only affecting the patient's quality of life, but also has a major impact on the healthcare services. Pregabalin is a first-line therapy in pDPN that offers adequate pain relief and is well tolerated. Moreover, it is also effective in treating the co-morbidities of pDPN, which include anxiety and sleep interference. Nonetheless, further studies are required to investigate the effectiveness and underlying mechanisms of the benefits of pregabalin on anxiety and sleep disorders and to determine the type of patient that benefits most from this therapy. We also provide an overview of the mechanisms by which GABAergic drugs work in pDPN and consider new emerging therapies in this area.

\section{INTRODUCTION}

Pregabalin was approved for the management of neuropathic pain in 2004 in Europe and the US. First synthesised in 1990 [1], pregabalin (3-isobutyl gamma amino butyric acid gamma-analogue) [2] is a GABAergic drug primarily used in the treatment of neuropathic pain and is approved for use in over 120 countries. Its use as an adjuvant anti-epileptic is generally limited [3], although it is approved for use in partial seizures [4]. In clinical practice it can be used to treat generalised anxiety disorder because of its anxiolytic properties [4], but is primarily prescribed for painful diabetic neuropathy (pDPN), post-herpetic neuralgia and radicular pain as well as fibromyalgia $[5,6]$. Pregabalin and gabapentin are considered first-line treatment in the majority of international clinical guidelines for pDPN and form a key part of its management. Five professional bodies have produced expert guidance on the management of pDPN [7-11] and pregabalin is recommended as first-line therapy in all five guidelines, whilst duloxetine is recommended as first line in four of the guidelines (except the American Academy of Neurology) [7-11]. The latter is driven by only one duloxetine trial, being graded as class 1 evidence, because completion rates of other trials are $<80 \%$ [10].

Pregabalin is structurally related to the inhibitory neurotransmitter GABA; however, its mechanism of action is distinct from GABAergic modulation and is yet to be fully elucidated. Pain relief usually occurs within 1 week of initiating therapy and is thought to be mediated via high-affinity binding to the alpha2-delta subunit $(\alpha 2 \delta)$ of voltage-gated calcium channels at the presynaptic terminals [12]. This results in modulation of the release of excitatory neurotransmitters such as glutamate [12] through the glutamate synthesising enzyme, branchedchain amino acid transaminase [13, 14].

Pregabalin's mechanism of action is similar to that of gabapentin; however, it has 2-4 times more potency and thus a lower dosing strategy is required [15]. Typically starting doses range from 75 to $150 \mathrm{mg}$ per day for neuropathic pain, with relatively quick up-titration over several weeks to maximal tolerated doses $(600 \mathrm{mg} /$ day) due to the more linear pharmacokinetics compared with gabapentin [15]. It has high bioavailability $(\geq 90 \%$ rapidly absorbed) $[16,17]$ with a half-life of approximately $9 \mathrm{~h}$ [18]. Very common adverse effects $(>10 \%$ of patients) include dizziness, somnolence and headache [17]. Pregabalin demonstrates quick absorption, reducing the probability of drugdrug interactions as it is not bound to plasma proteins and does not undergo first-pass metabolism in the liver $[4,19]$. Although pregabalin poses low risk for addiction and drug dependence at therapeutic doses [20], recent statistics 
in England and Wales showed a $>20$-fold increase in the number of deaths linked to pregabalin-4 to 111 deaths from 2012 to 2016, whilst for gabapentin-8 to 59 deaths during the same period [21]. Rapid rise in mortality is associated with individuals with previous history of recreational polydrug misuse, or misuse in combination with opioids, as some report self-administration of dosages in excess (e.g., up to 10-20 times) of clinically advisable dosages [22]. Consequently, this led to guidance from Public Health England and the NHS on safe prescribing of both pregabalin and gabapentin [23]. However, in December 2017 NHS England launched a consultation to seek views on whether to schedule pregabalin and gabapentin as Controlled drugs in the UK. A similar consultation is underway with the WHO and a decision is expected in early 2019. Due to their "risk of addiction, potential illegal diversion and medicinal misuse" by a minority, the reclassification of these drugs will have major repercussions on the many millions of people suffering from neuropathic pain, as it will restrict prescribing and also prevent them from acquiring the drug on a repeat prescription.

\section{METHODS}

A comprehensive literature review was undertaken, incorporating article searches in electronic databases (EMBASE, PubMed, OVID) and reference lists of relevant articles with the authors' expertise in pDPN. The key following keywords were utilised; 'painful diabetic neuropathy', 'diabetic neuropathy', 'sleep interference', 'anxiety' and 'depression' in combination with 'pregabalin'. This article is based on previously conducted studies and does not contain any studies with human participants or animals performed by any of the authors.

\section{pDPN Prevalence and the Need for Therapy}

Diabetes has reached epidemic proportions worldwide, with current International Diabetes Federation (IDF) estimates suggesting a prevalence of 425 million people worldwide in 2017, rising to 628 million by 2045 [24]. Epidemiological studies show DPN has a prevalence of $30-40 \%$ in patients with diabetes [25-27], which increases with the duration of disease, rising to approximately $50 \%$ in those patients who have had the disease for $>20$ years [26]. The symptoms can be varied with patients presenting with paraesthesia, weakness and pain, which may by burning, tingling or shooting in nature. Pain can have a negative impact on patient's quality of life and sleep. There are currently no FDA approved therapies to prevent, slow or arrest DPN, and management involves achieving good glycaemic control and targeting modifiable vascular risk factors to halt the progression alongside symptomatic treatment. The management of pDPN is key to improving quality of life and general well-being; however, therapeutic options in pDPN are often limited by side effects. Pregabalin has a higher potency than gabapentin thus requiring lower doses, recommended dose of up to 600 $\mathrm{mg}$ /day in management of pDPN and a superior adverse effect profile [28]. It is the only agent in the gabapentanoid class with a current label for the treatment of pDPN [29]. Indeed, pregabalin is the only agent other than duloxetine and tapentadol to have FDA approval for the treatment of neuropathic pain [29].

\section{Pharmacodynamics}

GABA is a major inhibitory neurotransmitter in the central nervous system exerting inhibitory control on the spinal dorsal horn [30]. It is released by inhibitory descending fibres and interneurons and binds to both the $\mathrm{GABA}_{\mathrm{A}}$ (via ionotropic) and $\mathrm{GABA}_{\mathrm{B}}$ (via metabotropic) receptors depressing neuronal excitation and reducing glutamate release from primary afferent fibres onto dorsal horn neurones [31]. Modulation of excitatory neurotransmitters such as glutamate [12] through the glutamate synthesising enzyme, branched-chain amino acid transaminase, is thought to play a possible role in pain in pDPN $[13,14]$. Loss of the GABAergic inhibitory process has been identified as an underlying mechanism of inflammatory and neuropathic pain [32]; however, the 
exact mechanism remains unclear. A reduction in the expression of the potassium chloride transporter (KCC2) with primary sensory neurones exhibiting a higher intracellular chloride level has also been proposed as a possible contributory mechanism [33].

Despite being developed as a mimetic of GABA, pregabalin is not believed to produce any of its therapeutic effects directly via the GABA receptor. A high affinity to the $\alpha_{2}-\delta$ subunit found in a number of voltage-gated calcium channels (VGCC) is considered its primary mechanism of action [34]. Indeed, genetic knock-out of $\alpha_{2}-\delta$ in animal models entirely negates the analgesic effects of pregabalin $[35,36]$.

\section{Absorption Distribution, Metabolism, Excretion and Interactions}

Pregabalin's bioavailability is over $90 \%$ and is dose independent [37]. Food does not affect pregabalin's overall exposure; however, it can reduce the rate of absorption with the content of the food having little effect on this [38, 39]. Maximal serum concentrations appear $0.7-1.3 \mathrm{~h}$ post-dose [39] with the serum half-life ranging from 4.6 to $6.8 \mathrm{~h}$ [39]. Over $90 \%$ of its excretion is via the kidney [40]. There are very few drug-drug interactions reported with pregabalin [37], which may be in part because it has no significant effects on the CYP450 family [19]. As such, the only major clinical factor relevant to dosing is renal function. The maximum dose of $600 \mathrm{mg}$ /day should only be used in patients with a creatinine clearance $(\mathrm{CrCl})>60 \mathrm{ml} / \mathrm{min}$. In patients whose $\mathrm{CrCL}$ is $15-30 \mathrm{ml} / \mathrm{min}$, the maximum dose should be reduced $150 \mathrm{mg}$ in 1-2 divided doses and when CrCL is $<15 \mathrm{ml} / \mathrm{min}$ this needs to be reduced further to a maximum of $75 \mathrm{mg}$ once daily. The indications, dosing, renal dosing, and minor and major side effects for pregabalin, gabapentin and the emerging GABAergic therapy, mirogabalin, are presented in Tables 1 and 2 .

\section{Adverse Effects}

The frequently reported side effects of pregabalin include somnolence, dizziness, peripheral oedema and weight gain, which are usually mild to moderate $[39,41,42]$. These effects are thought to be related to the drug's effects on neurotransmitter release and calcium currents. Pregabalin may cause adverse events on cognition and coordination [43]. In addition, treatment is associated with weight gain, which is in part dependent on the total drug dose, thus raising an issue that needs to be considered in treating patients with type 2 diabetes. There is a theoretical risk of worsening metabolic control with the associated weight gain from gabapentanoids; a pooled analysis of data from 11 double-blind randomised controlled trials (RCT) of pregabalin (150-600 mg/day) vs. placebo showed no clinically meaningful effects of pregabalin on glycaemic or lipid profiles in patients with pDPN, whilst data on weight were not presented [44]. The effects on weight should be considered when starting pregabalin as part of a personalised treatment approach.

\section{Efficacy of Pregabalin in pDPN: Evidence from RCTs}

RCTs of pregabalin in pDPN, including NCT number, primary outcome measures and study population details, are summarised in Table 3.

The efficacy of pregabalin in pDPN is proven through numerous RCTs (Table 3 ) and systematic reviews. Pregabalin is the first drug to receive an approved labelling from the Food and Drug Association (FDA) for the treatment of pDPN and post-herpetic neuralgia [45]. It is the recommended first-line treatment in all five major international clinical guidelines for pDPN [7-11]. In an early RCT of pregabalin in pDPN in 2004, Rosenstock et al. showed significant improvement in the mean pain scores, sleep interference, mood disturbance and tension anxiety over 8 weeks on pregabalin $300 \mathrm{mg} /$ day $(n=146)$ [46]. Pregabalin was well tolerated despite the mild-to-moderate adverse events of increased dizziness and somnolence [46]. A shorter 5-week double blind multi-centre RCT $(n=338)$ randomised patients to receive $150 \mathrm{mg} /$ day, $300 \mathrm{mg} /$ day or $600 \mathrm{mg} /$ day of pregabalin or placebo [47]. Participants in the $300 \mathrm{mg} /$ day and $600 \mathrm{mg} /$ day subgroups showed 
Table 1 Pregabalin, gabapentin doses, titration, side effects and major side effects

\begin{tabular}{|c|c|c|c|c|c|}
\hline & $\begin{array}{l}\text { Indications of use } \\
\text { other than pDPN }\end{array}$ & Dose in pDPN & $\begin{array}{l}\text { Renal } \\
\text { impairment }\end{array}$ & $\begin{array}{l}\text { Commonly } \\
\text { reported side } \\
\text { effects }\end{array}$ & Major side effects \\
\hline $\begin{array}{r}\text { Pregabalin } \\
\text { (Lyrica) }\end{array}$ & $\begin{array}{l}\text { General neuropathic } \\
\text { pain, fibromyalgia, } \\
\text { epilepsy, post- } \\
\text { operative pain, } \\
\text { generalised anxiety } \\
\text { disorder, post- } \\
\text { herpetic neuralgia }\end{array}$ & $\begin{array}{l}\text { Initial: } 75 \mathrm{mg} \\
\text { BID } \\
\text { Titration: } 150 \mathrm{mg} \\
\text { BID within } 1 \text { week } \\
\text { based on efficacy } \\
\text { and tolerability } \\
\text { Maximum dose: } \\
600 \mathrm{mg} \text { a day in } \\
\text { patients with CrCl } \\
\text { of } \geq 60 \mathrm{ml} / \mathrm{min}\end{array}$ & $\begin{array}{l}\mathrm{CrCl}<15 \mathrm{ml} / \\
\text { min: Initial } \\
\text { dose: } 25 \mathrm{mg} \\
\text { OD } \\
\text { Maintenance: } \\
25-75 \mathrm{mg} \mathrm{OD} \\
\text { CrCl } 15-30 \mathrm{ml} / \\
\text { min: initial dose: } \\
25-50 \mathrm{mg} \text { in } \\
1-2 \text { divided } \\
\text { doses } \\
\text { Maintenance: } \\
25-150 \mathrm{mg} / \text { day } \\
\text { in } 1-2 \text { divided } \\
\text { doses } \\
\text { CrCl } 30-60 \mathrm{ml} / \\
\text { min: initial dose: } \\
75 \text { mg/day in } 2 \\
\text { or } 3 \text { divided } \\
\text { doses } \\
\text { Maintenance: } \\
75-300 \mathrm{mg} / \text { day } \\
\text { in } 2-3 \text { divided } \\
\text { doses }\end{array}$ & $\begin{array}{l}\text { Drowsiness, } \\
\text { dizziness, } \\
\text { fatigue, ataxia, } \\
\text { headache, } \\
\text { blurred vision, } \\
\text { peripheral } \\
\text { oedema, } \\
\text { tremor, weight } \\
\text { gain }\end{array}$ & $\begin{array}{l}\text { Confusion, visual } \\
\text { disturbance, } \\
\text { abnormal gait, } \\
\text { abnormality in } \\
\text { thinking, amnesia, } \\
\text { vertigo }\end{array}$ \\
\hline $\begin{array}{l}\text { Gabapentin } \\
\text { (off-label) } \\
\text { (Neurontin, } \\
\text { Horizant, } \\
\text { Gralise) }\end{array}$ & $\begin{array}{l}\text { General neuropathic } \\
\text { pain, fibromyalgia, } \\
\text { epilepsy, restless } \\
\text { legs syndrome, pain } \\
\text { associated with } \\
\text { Guillain-Barré } \\
\text { syndrome, } \\
\text { phantom limb pain }\end{array}$ & $\begin{array}{l}\text { Neuropathic pain: } \\
\text { initial dose: } \\
300 \mathrm{mg} \text { day 1, } \\
300 \mathrm{mg} \text { BD day 2, } \\
300 \mathrm{mg} \text { TDS day } 3 \\
\text { Therapeutic dose: } \\
1800-3600 \mathrm{mg} / \text { day } \\
\text { in } 3 \text { divided doses in } \\
\text { patients with CrCl } \\
\geq 60 \mathrm{ml} / \mathrm{min} \\
\text { Maximum dose: } \\
3600 \mathrm{mg} / \text { day in } 3 \\
\text { divided doses }\end{array}$ & & $\begin{array}{l}\text { Mood or } \\
\text { behaviour } \\
\text { changes, } \\
\text { anxiety, panic } \\
\text { attacks, trouble } \\
\text { sleeping, } \\
\text { impulsive, } \\
\text { irritable, } \\
\text { agitated, } \\
\text { oedema }\end{array}$ & $\begin{array}{l}\text { Increased seizures, } \\
\text { severe weakness or } \\
\text { tiredness, ataxia, } \\
\text { upper GI pain, } \\
\text { severe tingling or } \\
\text { numbness, rapid } \\
\text { eye movement, } \\
\text { little or no } \\
\text { urination, oedema, } \\
\text { depression or } \\
\text { suicidal thoughts }\end{array}$ \\
\hline
\end{tabular}

$O D$ once daily, $B I D$ two times daily, $T D S$ three times daily 
Table 2 Emerging GABAergic therapies

\begin{tabular}{|c|c|c|c|c|c|}
\hline & Indication & Dose & $\begin{array}{l}\text { Renal } \\
\text { impairment }\end{array}$ & $\begin{array}{l}\text { Commonly reported side } \\
\text { effects }\end{array}$ & $\begin{array}{l}\text { Major side } \\
\text { effects }\end{array}$ \\
\hline Mirogabalin & $\begin{array}{l}\text { Peripheral neuropathy } \\
\text { fibromyalgia, post-herpetic } \\
\text { neuralgia (marketing } \\
\text { application submitted Feb. } \\
\text { 2018; approval/launch } \\
\text { planned for 2019) [117] }\end{array}$ & $\begin{array}{l}\text { Initial dose: } \\
15 \mathrm{mg} \text { OD } \\
\text { Maintenance } \\
\text { dose: } 15 \mathrm{mg} \\
\text { OD or BD } \\
\text { Maximum } \\
\text { dose: } \\
30 \mathrm{mg} / \text { day }\end{array}$ & $\begin{array}{l}\mathrm{CrCl} \\
15-29 \mathrm{ml} / \\
\mathrm{min}: \\
7.5 \mathrm{mg} \\
\mathrm{OD} \\
\mathrm{CrCl} \\
30-59 \mathrm{ml} / \\
\mathrm{min}: \\
7.5 \mathrm{mg} \mathrm{BD} \\
{[118]}\end{array}$ & $\begin{array}{l}\text { Somnolence, dizziness, } \\
\text { headache, balance } \\
\text { disorder, vomiting, } \\
\text { peripheral oedema, } \\
\text { fatigue, constipation, } \\
\text { decreased appetite }\end{array}$ & $\begin{array}{l}\text { Headache, } \\
\text { cardiac } \\
\text { conduction } \\
\text { abnormality, } \\
\text { arrhythmia }\end{array}$ \\
\hline
\end{tabular}

$O D$ once daily, $B I D$ two times daily

an improvement in mean pain score, sleep interference score, patient global impression of change, short-form (SF) McGill Questionnaire and multiple domains for the SF-36 Health Survey [47]. There was a $>50 \%$ reduction in pain compared with baseline in $45 \%$ on $300 \mathrm{mg} /$ day and $48 \%$ on $600 \mathrm{mg} /$ day of pregabalin compared with $18 \%$ on placebo [47]. In both studies, improvement in pain and sleep began at 1 week and continued throughout the titration and maintenance phase.

In a 6-week RCT $(n=246)$ of patients randomised to $150 \mathrm{mg} /$ day or $600 \mathrm{mg} /$ day or placebo [48], $600 \mathrm{mg} /$ day of pregabalin led to a reduction in the mean pain score to 4.3 (vs. 5.6 for placebo, $P=0.002$ ) [49]. There was an increase in the proportion of participants who had $\mathrm{a} \geq 50 \%$ reduction of the mean pain score from baseline $(39 \%$ vs. $15 \%$ for placebo, $P=0.002$ ). This trial did not show efficacy of $150 \mathrm{mg} /$ day of pregabalin compared with placebo. Furthermore, a multi-centre trial $(n=338)$ reported significant reduction in the mean pain score in two different titration regimes of pregabalin with both final doses being $600 \mathrm{mg}$ [49]. More recently, Toole et al. evaluated 395 patients over 12 weeks randomised to pregabalin $150 \mathrm{mg}, 300 \mathrm{mg}$ or $600 \mathrm{mg}$ or placebo [28]. Forty-six per cent of patients on $600 \mathrm{mg} /$ day report a $>50 \%$ improvement in mean pain score from baseline (vs. $30 \%$ in placebo, $P=0.036$ ) [28]. Pregabalin $600 \mathrm{mg} /$ day had superior efficacy; however, there was no significant benefit with $150 \mathrm{mg} /$ day or $300 \mathrm{mg} /$ day subgroups compared with placebo [28]. The authors suggest this finding may be a result of the larger placebo response in one of the countries that participated who represented $42 \%$ of the patients [28]. The number needed to treat (NNT) was 6.3, whilst the number needed to harm $(\mathrm{NNH})$ (discontinuation because of adverse events) was 10.3 for pregabalin $600 \mathrm{mg} /$ day [28]. Pooled data from a recent meta-analysis supported previous data showing that pregabalin was superior to placebo for improving mean pain scores [50]. A 50\% pain reduction was greater with pregabalin than placebo. Three studies compared pregabalin at lower doses versus higher doses $(600 \mathrm{mg} /$ day $)$ and the withdrawal rate was higher with pregabalin $600 \mathrm{mg} /$ day [51]. Overall, pregabalin was well tolerated despite an increased risk of adverse events which limit dose titration.

Gilron et al. evaluated pregabalin across a range of neuropathic pain conditions, not limited to pDPN [52]. This RCT $(n=256)$ allowed for the assessment of concomitant, potentially confounding analgesics with stable dosing, thus reducing bias [52]. Flexible-dose pregabalin was prescribed for 4 weeks and some participants continued treatment for a further 5 weeks [52]. Modest levels of analgesia were observed at all 
Table 3 Randomised controlled trials of pregabalin in patients with pDPN

\begin{tabular}{|c|c|c|c|c|c|}
\hline Publication(s) & Study title & NCT ID & No. patients randomised & $\begin{array}{l}\text { Primary outcome } \\
\text { measures/results }\end{array}$ & $\begin{array}{l}\text { Country (no. of study } \\
\text { centres) }\end{array}$ \\
\hline $\begin{array}{l}\text { Mu et al. } \\
\quad[119]\end{array}$ & $\begin{array}{l}\text { An 11-week randomised, } \\
\text { double-blind, multi- } \\
\text { centre, placebo- } \\
\text { controlled study to } \\
\text { evaluate the efficacy, } \\
\text { safety and tolerability of } \\
\text { pregabalin ( } 300 \\
\text { mg/day) using a fixed } \\
\text { dosing schedule in } \\
\text { treatment of subjects } \\
\text { with pain associated } \\
\text { with diabetic peripheral } \\
\text { neuropathy }\end{array}$ & $\begin{array}{l}\text { NCT0133 } \\
2149\end{array}$ & $\begin{array}{l}620 \text { participants; } \\
\text { pregabalin, } n=313 \\
\text { placebo, } n=307\end{array}$ & $\begin{array}{l}\text { Baseline MPS, change } \\
\text { from baseline in MPS at } \\
\text { end point (day 63/week } \\
9) \\
\text { Improvement in MPS with } \\
\text { pregabalin vs. placebo } \\
\text { was not significant } \\
(P=0.0559) \text {. Pregabalin } \\
\text { significantly improved } \\
\text { weekly MPS } \\
(P=0.0164) \\
\text { and } \geq 50 \% \text { responders } \\
\text { at end point } \\
(P=0.0384)\end{array}$ & $\begin{array}{l}\text { Multi-centre: Chinese } \\
\text { population } \\
30 \text { study centres in } \\
\text { China }\end{array}$ \\
\hline $\begin{array}{l}\text { Huffman et al. } \\
\qquad[120]\end{array}$ & $\begin{array}{l}\text { A phase 3B multi-centre, } \\
\text { double-blind, } \\
\text { randomised, placebo- } \\
\text { controlled cross-over } \\
\text { efficacy and safety study } \\
\text { of pregabalin in the } \\
\text { treatment of patients } \\
\text { with painful diabetic } \\
\text { peripheral neuropathy } \\
\text { and pain on walking }\end{array}$ & $\begin{array}{l}\text { NCT0147 } \\
4772\end{array}$ & $\begin{array}{l}203 \text { patients in } 2 \text {-period } \\
\text { crossover study } \\
\text { (pregabalin, } n=198 ; \\
\text { placebo, } n=186 \text { ) }\end{array}$ & $\begin{array}{l}\text { No statistically significant } \\
\text { treatment difference for } \\
\text { pregabalin vs. placebo, } \\
\text { mean DPN pain } \\
(P=0.0656) \text { and mean } \\
\text { DPN pain on walking } \\
(P=0.412)\end{array}$ & $\begin{array}{l}\text { Multi-centre, } 30 \text { study } \\
\text { centres in US, Czech } \\
\text { Republic, South } \\
\text { Africa and Sweden }\end{array}$ \\
\hline $\begin{array}{l}\text { Raskin et al. } \\
\quad[121]\end{array}$ & $\begin{array}{l}\text { A study of pregabalin in } \\
\text { the treatment of subjects } \\
\text { with painful diabetic } \\
\text { peripheral neuropathy } \\
\text { with background } \\
\text { treatment of NSAID for } \\
\text { other pain conditions }\end{array}$ & $\begin{array}{l}\text { NCT0145 } \\
5415\end{array}$ & $\begin{array}{l}154 \text { patients pregabalin to } \\
\text { placebo; } 147 \text { patients } \\
\text { placebo to pregabalin }\end{array}$ & $\begin{array}{l}\text { Weekly MPS at end point } \\
\text { (14 weeks) showed no } \\
\text { significant difference } \\
\text { between pregabalin and } \\
\text { placebo. Secondary end } \\
\text { point: mean treatment } \\
\text { difference in DPN- } \\
\text { related sleep } \\
\text { interference, favoured } \\
\text { pregabalin over placebo } \\
(P=0.0009)\end{array}$ & $\begin{array}{l}\text { Multi-centre } \\
47 \text { study centres in US } \\
\text { [43], Czech Republic } \\
\text { [3], Italy [1] }\end{array}$ \\
\hline
\end{tabular}


Table 3 continued

\begin{tabular}{|c|c|c|c|c|c|}
\hline Publication(s) & Study title & NCT ID & No. patients randomised & $\begin{array}{l}\text { Primary outcome } \\
\text { measures/results }\end{array}$ & $\begin{array}{l}\text { Country (no. of study } \\
\text { centres) }\end{array}$ \\
\hline $\begin{array}{l}\text { Raskin et al. } \\
\text { [122] }\end{array}$ & $\begin{array}{l}\text { A phase } 3 \mathrm{~B} \text { multi-centre, } \\
\text { double-blind, } \\
\text { randomised withdrawal } \\
\text { efficacy and safety study } \\
\text { of pregabalin in the } \\
\text { treatment of patients } \\
\text { with inadequately } \\
\text { treated painful diabetic } \\
\text { peripheral neuropathy }\end{array}$ & $\begin{array}{l}\text { NCT0105 } \\
7693\end{array}$ & $\begin{array}{l}665 \text { patients in 6-week } \\
\text { single-blind run-in } \\
\text { period (pregabalin } \\
300 \mathrm{mg} / \text { day); } 294 \\
\text { patients with }>30 \% \\
\text { pain response were } \\
\text { randomised to receive } \\
\text { pregabalin } \\
\text { (300 mg/day) or } \\
\text { placebo for a further } \\
12 \text { weeks }\end{array}$ & $\begin{array}{l}\text { In single-blind treatment } \\
\text { phase, MPS decreased } \\
\text { numerically. In double- } \\
\text { blind treatment phase, } \\
\text { MPS pregabalin group } \\
\text { decreased from } 6.8 \text { at } \\
\text { single-blind baseline to } \\
2.9(1.7) \text { at double-blind } \\
\text { end point, a change } \\
\text { from single-blind } \\
\text { baseline of - } 3.9 \text { (1.9). } \\
\text { MPS placebo group } \\
\text { decreased from } 6.7 \text { at } \\
\text { single-blind baseline to } \\
\text { 3.2 (1.9), a change from } \\
\text { single-blind phase of } \\
\text { - 3.5 (2.1); least squares } \\
\text { mean difference, } \\
-0.32) \text {, no significant } \\
\text { difference }\end{array}$ & $\begin{array}{l}\text { Multi-centre } \\
129 \text { study centres in the } \\
\text { US [113], South } \\
\text { Africa [11], Canada } \\
\text { [5] }\end{array}$ \\
\hline $\begin{array}{l}\text { Satoh et al. } \\
\quad[123]\end{array}$ & $\begin{array}{l}\text { Randomised, double-blind, } \\
\text { multi-centre, placebo- } \\
\text { controlled study to } \\
\text { evaluate efficacy and } \\
\text { safety of pregabalin (CI- } \\
\text { 1008) in the treatment } \\
\text { for pain associated with } \\
\text { diabetic peripheral } \\
\text { neuropathy }\end{array}$ & $\begin{array}{c}\text { NCT0055 } \\
3475\end{array}$ & $\begin{array}{l}317 \text { patients (placebo or } \\
\text { pregabalin at } 300 \text { or } \\
600 \mathrm{mg} / \text { day) }\end{array}$ & $\begin{array}{l}\text { Significant reductions in } \\
\text { pain with pregabalin at } \\
300 \text { and } 600 \mathrm{mg} / \text { day vs. } \\
\text { placebo, observed as } \\
\text { early as week } 1 \text { and } \\
\text { sustained throughout } \\
\text { study period (-0.63 } \\
\text { and }-0.74 \text {, } \\
\text { respectively) }\end{array}$ & $\begin{array}{l}\text { Multi-centre Japanese } \\
\text { population }\end{array}$ \\
\hline $\begin{array}{l}\text { Gilron et al. } \\
\text { [52] }\end{array}$ & $\begin{array}{l}\text { A randomised, placebo- } \\
\text { controlled trial of the } \\
\text { efficacy and safety of } \\
\text { pregabalin in the } \\
\text { treatment of subjects } \\
\text { with peripheral } \\
\text { neuropathic pain }\end{array}$ & $\begin{array}{l}\text { NCT0021 } \\
9544\end{array}$ & $\begin{array}{l}256 \text { patients in run-in } \\
\text { period; } 165(65 \%) \\
\text { had }>30 \% \text { pain } \\
\text { improvement, } 157 \text { were } \\
\text { randomised to either } \\
\text { pregabalin }(n=80) \text { or } \\
\text { to receive placebo } \\
(n=77)\end{array}$ & $\begin{array}{l}\text { At the double-blind end } \\
\text { point, MPS was } 2.9 \\
(1.9) \text { in pregabalin } \\
\text { group and } 3.5(1.7) \text { in } \\
\text { placebo group } \\
(P=0.002)\end{array}$ & $\begin{array}{l}\text { Multi-centre Canadian } \\
\text { population }[25]\end{array}$ \\
\hline
\end{tabular}


Table 3 continued

\begin{tabular}{|c|c|c|c|c|c|}
\hline Publication(s) & Study title & NCT ID & No. patients randomised & $\begin{array}{l}\text { Primary outcome } \\
\text { measures/results }\end{array}$ & $\begin{array}{l}\text { Country (no. of study } \\
\text { centres) }\end{array}$ \\
\hline $\begin{array}{l}\text { Guan et al. } \\
\quad[124]\end{array}$ & $\begin{array}{l}\text { An } 8 \text {-week multi-centre, } \\
\text { randomised, double- } \\
\text { blind, placebo- } \\
\text { controlled study to } \\
\text { evaluate the efficacy, } \\
\text { safety and tolerability of } \\
\text { pregabalin (150-600 } \\
\text { mg/day) using a flexible } \\
\text { dosing schedule in the } \\
\text { treatment of subjects } \\
\text { with symptoms of } \\
\text { neuropathic pain }\end{array}$ & $\begin{array}{l}\text { NCT0030 } \\
1223\end{array}$ & $\begin{array}{l}\text { Flexible-dose pregabalin } \\
150-600 \mathrm{mg} / \text { day. } \\
\text { Randomised in } 2: 1 \\
\text { ratio. Treatment } \\
\text { pregabalin }(n=206), \\
\text { placebo }(n=102)\end{array}$ & $\begin{array}{l}\text { MPS with pregabalin } \\
\text { resulted in significant } \\
\text { improvement compared } \\
\text { with placebo, a least } \\
\text { squares mean difference } \\
\text { score of }-0.6 \\
(P=0.005) ; 64 \% \text { and } \\
52 \% \text { patients treated } \\
\text { with pregabalin and } \\
\text { placebo, respectively, } \\
\text { reported } \geq 30 \% \\
\text { improvement in MPS } \\
(P=0.04)\end{array}$ & $\begin{array}{l}\text { Multi-centre } \\
\text { Chinese population }\end{array}$ \\
\hline $\begin{array}{l}\text { Parsons and } \mathrm{Li} \\
\quad[125]\end{array}$ & $\begin{array}{l}\text { A randomised, double- } \\
\text { blind, placebo- } \\
\text { controlled, parallel- } \\
\text { group, multi-centre trial } \\
\text { of pregabalin versus } \\
\text { placebo in the treatment } \\
\text { of neuropathic pain } \\
\text { associated with diabetic } \\
\text { peripheral neuropathy }\end{array}$ & $\begin{array}{l}\text { NCT0014 } \\
3156\end{array}$ & $\begin{array}{l}\text { Data pooled from } 11 \\
\text { placebo-controlled trials } \\
\text { to evaluate efficacy } \\
\text { pregabalin flexible or } \\
\text { fixed dose }(150,300 \text { or } \\
600 \mathrm{mg} / \text { day })\end{array}$ & $\begin{array}{l}\text { At baseline, } 1816 \text { patients } \\
\text { had moderate pain } \\
\text { (pregabalin, } n=1189 \text { ) } \\
\text { and } 1119 \text { patients had } \\
\text { severe pain (pregabalin, } \\
n=720 \text { ). Pregabalin } \\
(300,600 \mathrm{mg} / \text { day) } \\
\text { significantly reduced } \\
\text { MPS at end point } \\
\text { compared with placebo } \\
\text { ( } p<0.01 \text { ). Pregabalin } \\
\text { improved PRSI and } \\
\text { PGIC in moderate and } \\
\text { severe cohorts compared } \\
\text { with placebo }\end{array}$ & $\begin{array}{l}\text { Multi-centre pooled } \\
\text { data analysis }\end{array}$ \\
\hline $\begin{array}{l}\text { Freeman et al. } \\
\qquad[126]\end{array}$ & $\begin{array}{l}\text { A } 14 \text {-week, double-blind, } \\
\text { randomised, placebo- } \\
\text { controlled, multi-centre } \\
\text { study to evaluate the } \\
\text { safety and efficacy of } \\
\text { pregabalin (150-600 } \\
\text { mg/day) using a flexible } \\
\text { optimal dose schedule in } \\
\text { patients with painful } \\
\text { diabetic peripheral } \\
\text { neuropathy (DPN) }\end{array}$ & $\begin{array}{l}\text { NCT0015 } \\
6078\end{array}$ & $\begin{array}{l}\text { Data pooled from } 16 \\
\text { randomised, placebo- } \\
\text { controlled, parallel- } \\
\text { group, double-blind } \\
\text { trials of pregabalin for } \\
\text { treatment of } 3053 \\
\text { patients with DPN }\end{array}$ & $\begin{array}{l}\text { At baseline, MPS of } \geq 4, \\
\text { for } 4 \text { or more days prior } \\
\text { to randomisation. } \\
\text { Flexible dose pregabalin } \\
\text { (150-600 mg/day); no } \\
\text { significant difference in } \\
\text { MPS compared with } \\
\text { placebo (A0081030). } \\
\text { No significant difference } \\
\text { in MPS with pregabalin } \\
\text { (300 or } 600 \text { mg/day) } \\
\text { compared with placebo } \\
\text { (A0081071). No } \\
\text { significant difference in } \\
\text { MPS with pregabalin } \\
600 \text { mg/day } \\
\text { (1008-040) }\end{array}$ & $\begin{array}{l}\text { Multi-centre pooled } \\
\text { data analysis (19 } \\
\text { countries across Asia, } \\
\text { Europe, Latin } \\
\text { America and Middle } \\
\text { East, US) }\end{array}$ \\
\hline
\end{tabular}


Table 3 continued

\begin{tabular}{|c|c|c|c|c|c|}
\hline Publication(s) & Study title & NCT ID & No. patients randomised & $\begin{array}{l}\text { Primary outcome } \\
\text { measures/results }\end{array}$ & $\begin{array}{l}\text { Country (no. of study } \\
\text { centres) }\end{array}$ \\
\hline $\begin{array}{l}\text { Freeman et al. } \\
\qquad[127]\end{array}$ & $\begin{array}{l}\text { Meta-analysis: seven RCTs } \\
\text { across a range of } \\
\text { pregabalin doses }\end{array}$ & $\mathrm{n} / \mathrm{a}$ & $\begin{array}{l}1510 \text { patients in seven } \\
\text { double-blind RCTs: } \\
\text { pregabalin, } n=953 ; \\
\text { placebo, } n=557 . \text { MPS } \\
\text { baseline was } 6.5\end{array}$ & $\begin{array}{l}\text { MPS and PRSI score } \\
\text { improved associated } \\
\text { with pregabalin } 150 \text {, } \\
300 \text { and } 600 \mathrm{mg} / \text { day } \\
\text { administered TID vs. } \\
\text { placebo, } P<0.007\end{array}$ & $\begin{array}{l}\text { Multi-centre pooled } \\
\text { data analysis }(90 \% \\
\text { patients white, } 58 \% \\
\text { male) }\end{array}$ \\
\hline $\begin{array}{l}\text { Freynhagen } \\
\text { et al. [128] }\end{array}$ & $\begin{array}{l}\text { Comprehensive drug safety } \\
\text { evaluation of pregabalin } \\
\text { in } \mathrm{pDPN}^{* *}\end{array}$ & $\mathrm{n} / \mathrm{a}$ & $\begin{array}{l}7510 \text { patients included: } \\
4884 \text { on pregabalin and } \\
2626 \text { on placebo } \\
31 \text { RCTs of pregabalin in } \\
\text { pDPN }\end{array}$ & $\begin{array}{l}\text { Incidence of adverse } \\
\text { events: dizziness (risk } \\
\text { difference [95\% CI]: } \\
17.0 \text { [15.4 to } 18.6]) \text {, } \\
\text { somnolence (10.8 [9.5 } \\
\text { to } 12.1] \text { ), peripheral } \\
\text { oedema (5.4[4.3 to } \\
\text { 6.4]), weight increase } \\
\text { (4.7 [3.9 to 5.5]), dry } \\
\text { mouth (2.9 [2.1 to 3.8]), } \\
\text { constipation (2.3[1.5 to } \\
3.2]), \text { blurred vision }(2.2 \\
\text { [1.6 to 2.9]), balance } \\
\text { disorder (2.0 [1.5 to 2.5] } \\
\text { and euphoric mood (1.6 } \\
\text { [1.2 to } 2.0] \text { ) }\end{array}$ & $\begin{array}{l}\text { Multi-centre pooled } \\
\text { analysis RCT in Asia, } \\
\text { Australia, Canada, } \\
\text { Europe, Latin } \\
\text { America, the Middle } \\
\text { East, South Africa } \\
\text { and the US }\end{array}$ \\
\hline $\begin{array}{l}\text { Semel et al. } \\
\text { [129] }\end{array}$ & $\begin{array}{l}\text { Evaluation of safety and } \\
\text { efficacy of pregabalin in } \\
\text { older patients with } \\
\text { neuropathic pain: results } \\
\text { from a pooled analysis } \\
\text { of } 11 \text { clinical studies }\end{array}$ & $\mathrm{n} / \mathrm{a}$ & $\begin{array}{l}2516 \text { patients (white, } \\
\begin{aligned} n & =2344 ; \text { male } \\
n & =1347): \text { pregabalin, } \\
n & =1595\end{aligned}\end{array}$ & $\begin{array}{l}\text { MPS end point } \\
\text { improvement observed } \\
\text { for all pregabalin } \\
\text { dosages } \\
(150-600 \mathrm{mg} / \text { day }) \text { in } \\
\text { older patients } \\
\text { (age } \geq 65 \text { years old }) \\
(p \leq 0.0009)\end{array}$ & $\begin{array}{l}\text { Multi-centre (white } \\
\text { predominant) }\end{array}$ \\
\hline $\begin{array}{l}\text { Moon et al. } \\
\quad[130]\end{array}$ & $\begin{array}{l}\text { A 10-week, randomised, } \\
\text { double-blind, placebo- } \\
\text { controlled, multi-centre } \\
\text { study to evaluate the } \\
\text { efficacy and safety of } \\
\text { pregabalin (150-600 } \\
\text { mg/day) using a flexible, } \\
\text { optimised dose schedule } \\
\text { in subjects with } \\
\text { peripheral neuropathic } \\
\text { pain }\end{array}$ & $\begin{array}{l}\text { NCT0014 } \\
1219\end{array}$ & $\begin{array}{l}240 \text { patients. Phase III } \\
\text { trial. } 2: 1 \text { ratio to } \\
\text { pregabalin } \\
(150-600 \mathrm{mg} / \text { day }) \text { or } \\
\text { placebo. Pregabalin, } \\
n=162 \text {, placebo, } \\
n=78\end{array}$ & $\begin{array}{l}\text { MPS significantly lower in } \\
\text { pregabalin group than in } \\
\text { placebo; least square } \\
\text { mean difference, } \\
-0.50 ;-1.00 \text { to } 0.00 \text {; } \\
P=0.049 \text { ) }\end{array}$ & Korean population \\
\hline
\end{tabular}


Table 3 continued

\begin{tabular}{|c|c|c|c|c|c|}
\hline Publication(s) & Study title & NCT ID & No. patients randomised & $\begin{array}{l}\text { Primary outcome } \\
\text { measures/results }\end{array}$ & $\begin{array}{l}\text { Country (no. of study } \\
\text { centres) }\end{array}$ \\
\hline $\begin{array}{l}\text { Arezzo et al. } \\
\quad[131]\end{array}$ & $\begin{array}{l}\text { A } 13 \text {-week, double-blind, } \\
\text { placebo-controlled } \\
\text { phase } 4 \text { trial of } \\
\text { pregabalin (CI-1008, } \\
600 \mathrm{mg} \text { /day) for relief } \\
\text { of pain in subjects with } \\
\text { painful diabetic } \\
\text { peripheral neuropathy }\end{array}$ & $\begin{array}{l}\text { NCT0015 } \\
9679\end{array}$ & $\begin{array}{l}167 \text { patients (pregabalin, } \\
\begin{aligned} n & =82, \text { placebo, } \\
n & =85)\end{aligned}\end{array}$ & $\begin{array}{l}\text { MPS lower than placebo } \\
\text { (mean difference } \\
\quad-1.28 ; P<0.001 \text { ) }\end{array}$ & $\begin{array}{l}\text { US } \\
23 \text { study centres }\end{array}$ \\
\hline $\begin{array}{l}\text { Freynhagen } \\
\text { et al. [49] }\end{array}$ & $\begin{array}{l}\text { Efficacy and safety of } \\
\text { pregabalin using an } \\
\text { individual optimal twice } \\
\text { a day (BID) dose } \\
\text { schedule in patients } \\
\text { with chronic } \\
\text { neuropathic pain }\end{array}$ & $\mathrm{n} / \mathrm{a}$ & $\begin{array}{l}338 \text { patients randomised } \\
\text { in 1:2:2 ratio to placebo, } \\
\text { flexible-dose pregabalin } \\
\text { (150-600 mg/day) or } \\
\text { fixed dose pregabalin } \\
\text { ( } 600 \mathrm{mg} / \text { day). PGB } \\
\text { flexible dose, } n=141 ; \\
\text { PGB fixed dose, } \\
n=132 ; \text { placebo, } \\
n=65\end{array}$ & $\begin{array}{l}\text { MPS significantly reduced } \\
\text { for both flexible- and } \\
\text { fixed-dose pregabalin } \\
\text { versus placebo } \\
(P=0.002) \text { and PRSI } \\
\text { significantly improved }\end{array}$ & $\begin{array}{l}\text { European } \\
60 \text { study centres across } 9 \\
\text { EU countries }\end{array}$ \\
\hline $\begin{array}{l}\text { Tolle et al. } \\
2012[132]\end{array}$ & $\begin{array}{l}\text { A } 12 \text {-week, randomised, } \\
\text { double-blind, multi- } \\
\text { centre, placebo- } \\
\text { controlled study of } \\
\text { pregabalin twice a day } \\
\text { (BID) for relief of pain } \\
\text { associated with diabetic } \\
\text { peripheral neuropathy }\end{array}$ & $\mathrm{n} / \mathrm{a}$ & 395 participants & $\begin{array}{l}\text { MPS improved in } \\
\text { participants receiving } \\
\text { pregabalin } 600 \mathrm{mg} / \text { day; } \\
46 \% \text { reported }>50 \% \\
\text { improvement MPS } \\
\text { from baseline vs. } \\
\text { placebo patients } \\
\text { ( } P=0.036 \text { ). NNT was } \\
\text { 6.3. Improvement in } \\
\text { EQ-5D utility scores (all } \\
\text { pregabalin doses vs. } \\
\text { placebo. Number } \\
\text { needed to harm } \\
\text { (discontinuation } \\
\text { because of adverse } \\
\text { events) was } 10.3 \text { for } \\
\text { pregabalin } 600 \mathrm{mg} / \text { day }\end{array}$ & $\begin{array}{l}\text { European } \\
58 \text { study centres in } \\
\text { Europe (Germany, } \\
\text { Hungary, Poland and } \\
\text { the UK), Australia } \\
\text { and South Africa }\end{array}$ \\
\hline
\end{tabular}

NCT ID clinicaltrials.gov registry number, LOCF last observation carried forward, MPS mean pain score; DPRS = daily pain rating scale, PRSI pain-related sleep interference, $P G I C$ patient global impression of change, $N N T$ number needed to treat 
stages and at the final end point [52]. This was found to be greater in the pregabalin cohort compared with placebo in both pDPN and postherpetic neuralgia [52].

In clinical practice, lack of dose titration is common in primary care resulting in inadequate pain relief. Physicians treating patients with pDPN should escalate pregabalin to the dose that delivers optimal analgesia and tolerable side effects [53].

\section{Head-to-Head Trials of Comparators, Combination Therapy and Meta-Analyses}

In head-to-head studies of active comparators in pDPN, Bansal et al. [54] found no difference in the outcomes between amitriptyline and pregabalin. However, there was a greater proportion of adverse events in the amitriptyline group, $65 \%$ compared with $25 \%$ for pregabalin, and the preferred dose of pregabalin was $150 \mathrm{mg}$ BID. A further RCT comparing pregabalin, amitriptyline and duloxetine found no single treatment was superior [55]. Pregabalin was found to improve sleep continuity, whereas duloxetine increased wake and reduced total sleep time [55]. There were significantly more adverse events in the pregabalin group. A metaanalysis by Quilici et al. [56] (funded by Eli Lilly) determining the comparative efficacy of duloxetine vs. pregabalin and gabapentin through an indirect analysis found that all were superior to placebo for all efficacy parameters, with some tolerability trade-offs. Indirect comparison of duloxetine with pregabalin found no differences in 24-h average pain score but significant differences in patient global impression outcomes, favouring pregabalin, and in dizziness, favouring duloxetine [56]. The estimated NNT for duloxetine was 5 (95\% CI: 3-7) [56], which was comparable to NNTs reported elsewhere of 5.2 (95\% CI: 3.7-8.5) and 4.1 (95\% CI: 2.9-7.2) $[8,57]$ and to the NNT for pregabalin of 5 (95\% CI: 4-8). The NNH for pregabalin was 19 (95\% CI: 10-48) compared with duloxetine with an NNH of 11 (95\% CI: 7-23) [56]. In a study conducted in Spain (and funded by Pfizer) of pregabalin versus usual care (antidepressants, opioids, anticonvulsants different from pregabalin) in the management of communitytreated patients with refractory pDPN, pregabalin was associated with a non-significant higher quality-adjusted life-year (QALY) gain in a 12-week period [58]. In a further study by the same group, compared with gabapentin, pregabalin yielded an estimated mean of 8 [standard error (SE): 0.4] additional days with no or mild pain, 6 (SE: 0.4) days with $\geq 30 \%$ reduction in pain intensity, $9(0.5)$ days with $\geq 50 \%$ reduction in pain intensity and a gain of 0.1186 (0.0002) QALYs for 12 weeks [59]. Although the average study drug cost was higher for pregabalin than gabapentin (€214.6 vs. €157.4: $P<0.001)$, there was a lower cost of concomitant analgesic medication ( $€ 176.5$ vs. $€ 306.7$; $P<0.001$ ) [59].

The complex nature of $\mathrm{pDPN}$ may require more than one therapy for adequate pain relief, but there are limited studies of combination therapies [60]. The COMBO-DN study is the largest trial to date comparing monotherapy with a combination duloxetine and pregabalin in 339 participants [61]. There was no difference between combination standard-dose and highdose monotherapy of either treatment [61]. In a secondary analysis, duloxetine $60 \mathrm{mg}$ was found to be superior to pregabalin $300 \mathrm{mg}$ /day in the initial 8-week run-in phase. A further exploratory post hoc analysis of COMBO-DN showed that high-dose monotherapy was more favourable in patients with severe pain, whereas combination therapy was more beneficial in patients with moderate and mild pain [62]. Also, patients who received duloxetine (60 mg/day) as initial therapy had a better response to combined duloxetine and pregabalin for evoked or severe tightness and a greater benefit with high-dose duloxetine $(120 \mathrm{mg} /$ day $)$ for paraesthesia-dysaesthesia $[62,63]$. In another double-blind RCT with a parallel-group design comparing amitriptyline, duloxetine and pregabalin there was no significant difference in analgesic efficacy [64]. However, when determining polysomnographic parameters, pregabalin improved sleep continuity, whereas duloxetine increased wake and reduced total sleep time [64]. Despite the putative negative effects on sleep, duloxetine enhanced central nervous system arousal and 
performance on sensory motor tasks; however, there were significantly more adverse events in the pregabalin group [64].

A study of pregabalin and 5\% lidocaine medicated plaster $(n=229)$ in post-herpetic neuralgia or painful DPN [65] showed that patients who failed to respond to monotherapy of pregabalin gained additional benefit from the $5 \%$ lidocaine patch. This was in contrast to an RCT of the addition of low-dose $10 \mathrm{mg}$ oxycodone or placebo in patients treated with pregabalin where there was no enhancement of the pain-relieving effects of pregabalin [66].

\section{Anxiety, pDPN and Pregabalin}

There is a well-recognised triad of chronic pain, anxiety/depression and sleep interference, which impairs the activities of daily living in pDPN [67]. pDPN has a substantial impact on quality of life $[68,69]$, with $50-70 \%$ of patients attending chronic pain clinics reporting sleep impairment [70-72], > 20\% having major depression [73-76] and epidemiological data demonstrating excess anxiety levels compared with pain-free populations [77].

Pregabalin is approved in the European Union for use as an anxiolytic agent in the management of generalised anxiety disorder (GAD) and is commonly used-off label in the US [78]. With a mechanism of action distinct from other anxiolytic agents, it also offers broadspectrum treatment for the characteristic psychosomatic symptoms of GAD including excessive generalised worry, hypervigilance and persistent nonspecific anxiety [5]. Therefore, pregabalin is beneficial as a therapy in both pDPN and anxiety disorders, as a therapy in itself or as an adjunct.

Numerous studies have demonstrated pregabalin maintains improvements in anxiety symptoms in the long term and increases the time to GAD compared with placebo [5]. A retrospective cohort study [79] of three comparable 13-16-week RCTs [42, 80, 81] with their corresponding 52-week extensions in a Japanese population evaluated the use of various pregabalin regimens in pDPN, spinal cord injury (SCI) and post-herpetic neuralgia. Significant improvements in pain and sleep interference were seen after 1 week and subsequently maintained across all conditions including pDPN compared with placebo. At the end of the study, the least-squares (LS) mean pain scores (LOCF) were significantly reduced with pregabalin in all three trials. In the SCI trial, a non-significant difference in comparison with placebo was observed for the HADS anxiety and depression subscale scores in the pregabalin-treated group [79]. On completion of the studies significantly more patients treated with pregabalin, experienced a pain reduction $\geq 30 \%$ across all RCTs $[42,80,81]$. An extension of all three trials additionally demonstrated that reductions in pain intensity were maintained over a 12 -month period $[42,80,81]$. There were also notable improvements in 6 of the 16 SF-36 subscale scores in the DPN trial; the analgesic efficacy of pregabalin are similar across multiple neuropathic pain conditions and also improves quality-of-life measures and anxiety [79].

Pregabalin in the dose range $200-600 \mathrm{mg}$ /day (in two or three daily divided doses) significantly reduces mean pain scores on the Hamilton Anxiety Scale for GAD and social anxiety disorder (SAD) $[5,51]$. However, treatment-related adverse effects occurred in up to $50 \%$ of cases, of which somnolence precipitated study withdrawal for $\sim 32 \%$ patients [51]. An open-label, non-comparative, flexible-dose study $(n=217)$ in pDPN or post-herpetic neuralgia similarly investigated the correlation of patient and physician general global impression of change (patient global impression of change (PGIC) and clinician global impression of change (CGI)) with changes in pain, sleep and anxiety score as primary outcomes assessed on visual or numeric scales [82]. This multi-centre study identified significant improvements in pain, anxiety and sleep $(-40 \%,-42 \%,-43 \%$, respectively) when treated with pregabalin over 4 weeks from baseline to the end of the study [82]. The mean dose was $\sim 300 \mathrm{mg} /$ day but a limitation of these findings is the variable dosing regimen used [82]. Nonetheless, both pain and anxiety correlated with PGIC and CIGC and all correlations except paired CGIC/anxiety were statistically significant [82]. 


\section{Sleep Interference and Chronic Pain}

pDPN is associated with considerable sleep impairment, which has been highlighted as an important outcome measure [83, 84]. Sleep disturbance is associated with lower pain thresholds [85]. Pain sensitivity follows a diurnal pattern in keeping with a circadian variability with the variability of pain sensitivity increasing in response to the build-up of sleep pressure following sleep deprivation or disruption [86]. This variability has been further demonstrated in untreated pDPN and postherpetic neuralgia in two separate double-blind randomised controlled crossover trials where a relative pain intensity increase of $33 \%$ between 8 a.m. and 8 p.m. was reported [87-89].

\section{The Locus Coeruleus: A Centre for Pain and Sleep Mediation}

The locus coeruleus (LC) is a cluster of noradrenergic neurones in the dorsal pons with widespread projections throughout the brain [90]. The activity of the locus coeruleus peaks in wakefulness, declines during NREM sleep and is at its lowest during rapid eye movement (REM) sleep [90]. The LC has a complex role in neuropathic pain modulation as it may facilitate as well as inhibit pain development and maintenance of allodynia and hyperalgesia after nerve injury [91]. Studies limited to animal models have shown increased activity of the LC via surrogate markers of gene expression, which was directly proportional to the degree of allodynia [92]. Furthermore, an agonistic and analgesic effect was demonstrated when substance P was injected directly into the LC [93]. However, this effect was negated by prior injection of a neurokinin-1 receptor antagonist (the functional binding site of substance-P) and another agent, yohimbine, an alpha-2 adrenergic receptor antagonist [94]. Gabapentin demonstrates an 'anti-hypersensitivity' effect thought to be a direct effect on the LC in male rats in a peripheral nerve injury model, which shows increased LC activity [95].

\section{Pregabalin, pDPN and Sleep}

Pregabalin has shown considerable efficacy for improved sleep interference as demonstrated by an improvement in sleep quality in $77 \%$ of patients [96]. Pregabalin and gabapentin are effective in treating neuropathic pain and have a positive effect on co-morbid sleep disturbance compared with opiates and antidepressants [97]. Sleep disturbance as well as its severity correlates with the severity of neuropathic pain and is a predictor of response to pregabalin in a post hoc analysis of placebo-controlled trials [98]. Pregabalin significantly reduced pain scores and the greatest reduction was seen in those with the severest indices of sleep disturbance at baseline [98].

In a systematic review of nine clinical trials, pregabalin was found to be an effective and well-tolerated therapy to reduce pain and painrelated sleep disturbance in pDPN and postherpetic neuralgia [99]. It is important to note, however, that none of these studies reported objective sleep measures [99].

\section{Phantom Limb and Pregabalin}

Diabetes is the most common cause of nontraumatic lower limb amputation, with diabetic foot ulcers preceding $>80 \%$ of amputations in people with diabetes [100]. Phantom limb pain (PLP) can affect up to $80 \%$ of amputees [101] and is associated with considerable distress to patients and their carers. Pain often commences shortly after surgery once the initial surgical insult to the residual stump has passed. The phantom pain is described as shooting, stabbing, throbbing and/or burning, which often disturbs sleep [102]. It is associated with increased stress, anxiety, depression and reduced quality of life [103]. Mobilisation can be delayed whilst pain control is established, thus reducing the post-operative rehabilitation phase [104].

There remains a paucity of data in the use of pregabalin in PLP [105]. Currently, pre-emptive use of pregabalin for PLP is sporadic within the UK. Additional evidence is required in the form of well-designed and adequately powered RCTs 
to ascertain the role of pregabalin in standard of care of amputees and PLP. Indeed, a systematic review of original research studies specifically investigating the pharmacological treatment of PLP suggested that gabapentin had a higher level of evidence than pregabalin [105].

\section{Novel Therapies for $\mathrm{pDPN}$}

Voltage-sensitive calcium channels contain subunits alpha-2-delta $1\left(\alpha_{2} \delta-1\right)$ and alpha-2delta $2\left(\alpha_{2} \delta-2\right)$ subunits. $\alpha_{2} \delta-1$ interacts with NMDA receptors and promotes synaptic expression of $\alpha_{2} \delta$-1-NMDA receptor complexes in neuropathic pain [106]. Gabapentin and pregabalin reduce neuropathic pain by non-selectively targeting the $\alpha_{2} \delta$-1 subunit bound to NMDA receptors [107], inhibiting release of neurotransmitters such as glutamate and reducing hyperexcitability at the spinal cord. Mirogabalin (DS-5565) (Daiichi-Sankyo, Japan) has demonstrated higher affinity for the $\alpha_{2} \delta-1$ [108]. Vinik et al. (2014) conducted a large multi-centre, phase 2 RCT incorporating an active comparator group with dose-ranging mirogabalin therapy $(5,10,15,20,30 \mathrm{mg}$ /day) compared with pregabalin and placebo [109]. An early reduction of average daily pain scores, sustained after 5 weeks of therapy, was reported with mirogabalin 15, 20 and $30 \mathrm{mg}$ /day relative to placebo. In comparison, participants on pregabalin $300 \mathrm{mg} /$ day reported no significant difference in pain reduction compared with placebo at the end of a 5-week treatment period. The most common adverse effects were dizziness $(9.4 \%)$, somnolence $(6.1 \%)$ and headache (6.1\%) [109].

Voltage-gated sodium (Na) channels have emerged as promising therapeutic targets selectively targeting $\mathrm{Na}_{\mathrm{v}}$ channels. In particular, $\mathrm{Na}_{\mathrm{v}} 1.7$ has attracted the most attention as humans with $\mathrm{Na}_{\mathrm{v}} 1.7$ gain-of-function mutations suffer severe chronic pain syndromes [110]. Numerous trials are ongoing for $\mathrm{Na}_{\mathrm{v}}$ channel antagonists. There are ongoing studies by Pfizer (PF-05089771) [111], entering phase II trials in DPN), Biogen (BIIB074, previously known as CNV-1014802) [112], Xenon and Teva (XEN-402 or TV-45070; phase II in DPN) [113], Sumitomo Dainippon Pharma (DSP-2230; phase I trial) [114] and AstraZeneca (AZD-3161; phase II trial, GlaxoSmithKline (GSK-2339345) phase II trial) [115] and Gilead (GS-6615) [116].

\section{CONCLUSION}

pDPN significantly impacts the individual's quality of life, posing threats of chronic pain, sleep and mood disturbance. Management of the debilitating burden of pDPN thus requires symptomatic relief via well-researched pharmacotherapy such as pregabalin. Recommended as first line in the majority of guidelines, pregabalin is established as an effective and relatively well-tolerated agent, most useful in alleviating subjective sleep and mood disturbance. Pregabalin has good efficacy for treating pDPN and an adequate safety profile. Typically reported adverse effects of dizziness and somnolence prevent up-titration to the maximum of $600 \mathrm{mg} /$ day. However, several randomised-controlled trials have demonstrated symptomatic improvement in pain and sleep disturbance being achieved at lower doses (300 mg/day). Despite numerous large multi-centre RCTs comparing pregabalin with placebo, the average follow-up duration is relatively short, averaging at 6 weeks up to the longest trial duration of 14 weeks. Longer follow-up duration would be necessary to further evaluate and recognise the potential for sustained benefits and tolerability, although this should not negatively impact its use as this is on par with other trials for treatments of pDPN.

\section{ACKNOWLEDGEMENTS}

Funding. No funding or sponsorship was received for this study or publication of this article. 
Authorship. All named authors meet the International Committee of Medical Journal Editors (ICMJE) criteria for authorship for this article, take responsibility for the integrity of the work as a whole and have given their approval for this version to be published.

Disclosures. Uazman Alam has received honoraria for educational meetings from Pfizer and Eli Lilly and is currently a local investigator for BIIB074 (Biogen: NCT03339336). Rayaz A. Malik has received honoraria for educational meetings from Pfizer and Eli Lilly. Shazli Azmi has received honoraria for educational meetings from Eli Lilly. Kariem T. ElHadd, Andrew Nelson, Adam Chapman, Frank L. Bowling, Anughara Perumbalath, Jonathan Lim and Andrew Marshall have nothing to disclose.

Compliance with Ethics Guidelines. This article is based on previously conducted studies and does not contain any studies with human participants or animals performed by any of the authors.

Data Availability. No new data were generated during this narrative review of published studies.

Open Access. This article is distributed under the terms of the Creative Commons Attribution-NonCommercial 4.0 International License (http://creativecommons.org/licenses/ by-nc/4.0/), which permits any noncommercial use, distribution, and reproduction in any medium, provided you give appropriate credit to the original author(s) and the source, provide a link to the Creative Commons license, and indicate if changes were made.

\section{REFERENCES}

1. Andruszkiewicz R, Silverman RB. 4-Amino-3alkylbutanoic acids as substrates for gammaaminobutyric acid aminotransferase. J Biol Chem. 1990;265(36):22288-91.

2. Pritchard N, Edwards K, Russell AW, Perkins BA, Malik RA, Efron N. Corneal confocal microscopy predicts 4-year incident peripheral neuropathy in type 1 diabetes. Diabetes care. 2015:dc142114.

3. Toth C. Pregabalin: latest safety evidence and clinical implications for the management of neuropathic pain. Ther Adv Drug Saf. 2014;5(1):38-56.

4. D'Arcy Y, McCarberg B, Parsons B, Behar R, Thorpe A, Alexander A. Pregabalin for the treatment of neuropathic pain: a narrative review for primary care providers. Curr Med Res Opin. 2017;33(8):1353-9.

5. Frampton JE. Pregabalin: a review of its use in adults with generalized anxiety disorder. CNS Drugs. 2014;28(9):835-54.

6. Tavakoli M, Mitu-Pretorian M, Petropoulos IN, Fadavi H, Asghar O, Alam U, et al. Corneal confocal microscopy detects early nerve regeneration in diabetic neuropathy after simultaneous pancreas and kidney transplantation. Diabetes. 2012:DB_120574.

7. Handelsman Y, Bloomgarden ZT, Grunberger G, Umpierrez G, Zimmerman RS, Bailey TS, et al. American Association of Clinical Endocrinologists and American College of Endocrinology-Clinical Practice Guidelines for Developing a Diabetes Mellitus Comprehensive Care Plan-2015-executive summary. Endocr Pract. 2015;21(4):413-37.

8. Attal N, Cruccu G, Baron R, Haanpaa M, Hansson P, Jensen TS, et al. EFNS guidelines on the pharmacological treatment of neuropathic pain: 2010 revision. Eur J Neurol. 2010;17(9):1113-e88.

9. Bril V, England J, Franklin GM, Backonja M, Cohen J, Del Toro D, et al. Evidence-based guideline: treatment of painful diabetic neuropathy: report of the American Academy of Neurology, the American Association of Neuromuscular and Electrodiagnostic Medicine, and the American Academy of Physical Medicine and Rehabilitation. Neurology. 2011;76(20):1758-65.

10. Pop-Busui R, Boulton AJ, Feldman EL, Bril V, Freeman R, Malik RA, et al. Diabetic neuropathy: a position statement by the American Diabetes Association. Diabetes Care. 2017;40(1):136-54.

11. NICE. Neuropathic pain in adults: pharmacological management in non-specialist settings. NICE. 2017.

12. Fink K, Dooley DJ, Meder WP, Suman-Chauhan N, Duffy S, Clusmann $\mathrm{H}$, et al. Inhibition of neuronal $\mathrm{Ca}(2+)$ influx by gabapentin and pregabalin in the human neocortex. Neuropharmacology. 2002;42(2):229-36.

13. Hutson SM, Berkich D, Drown P, Xu B, Aschner M, LaNoue KF. Role of branched-chain 
aminotransferase isoenzymes and gabapentin in neurotransmitter metabolism. J Neurochem. 1998;71(2):863-74.

14. Micheva KD, Taylor CP, Smith SJ. Pregabalin reduces the release of synaptic vesicles from cultured hippocampal neurons. Mol Pharmacol. 2006;70(2):467-76.

15. Moore RA, Straube S, Wiffen PJ, Derry S, McQuay HJ. Pregabalin for acute and chronic pain in adults. Cochrane Database Syst Rev. 2009;3:Cd007076.

16. Lauria-Horner BA, Pohl RB. Pregabalin: a new anxiolytic. Expert Opin Investig Drugs. 2003;12(4):663-72.

17. Azmi S, Ferdousi M, Petropoulos IN, Ponirakis G, Fadavi H, Tavakoli $M$, et al. Corneal confocal microscopy shows an improvement in small-fiber neuropathy in subjects with type 1 diabetes on continuous subcutaneous insulin infusion compared with multiple daily injection. Diabetes Care. 2015;38(1):e3-4.

18. Randinitis EJ, Posvar EL, Alvey CW, Sedman AJ, Cook JA, Bockbrader HN. Pharmacokinetics of pregabalin in subjects with various degrees of renal function. J Clin Pharmacol. 2003;43(3):277-83.

19. Bockbrader HN, Wesche D, Miller R, Chapel S, Janiczek N, Burger P. A comparison of the pharmacokinetics and pharmacodynamics of pregabalin and gabapentin. Clin Pharmacokinet. 2010;49(10):661-9.

20. Schifano F. Misuse and abuse of pregabalin and gabapentin: cause for concern? CNS Drugs. 2014;28(6):491-6.

21. Statistics OfN. Deaths related to drug poisoning in England and Wales: 2016 registrations 2017. https://www.ons.gov.uk/peoplepopulationandcom munity/birthsdeathsandmarriages/deaths/bulletins/ deathsrelatedtodrugpoisoninginenglandandwales/ 2016registrations-people-aged-40-to-49-years-havethe-highest-rate-of-drug-misuse-deaths.

22. Iacobucci G. UK government to reclassify pregabalin and gabapentin after rise in deaths. BMJ. $2017 ; 358$.

23. Stannard C, Brew I, Browne E, Brinksman S, Collett B, Gilvarry E, et al. Advice for prescribers on the risk of the misuse of pregabalin and gabapentin. Public Health England and NHS England; 2014:pp 1-9.

24. International Diabetes Federation. IDF Diabetes Atlas. 8th ed. Brussels: International Diabetes Federation; 2017.
25. Gordois A, Scuffham P, Shearer A, Oglesby A, Tobian JA. The health care costs of diabetic peripheral neuropathy in the US. Diabetes Care. 2003;26(6):1790-5.

26. Van Acker K, Bouhassira D, De Bacquer D, Weiss S, Matthys K, Raemen H, et al. Prevalence and impact on quality of life of peripheral neuropathy with or without neuropathic pain in type 1 and type 2 diabetic patients attending hospital outpatients clinics. Diabetes Metab. 2009;35(3):206-13.

27. Boulton AJ, Vinik AI, Arezzo JC, Bril V, Feldman EL, Freeman R, et al. Diabetic neuropathies a statement by the American Diabetes Association. Diabetes Care. 2005;28(4):956-62.

28. Tolle T, Freynhagen R, Versavel M, Trostmann U, Young JP Jr. Pregabalin for relief of neuropathic pain associated with diabetic neuropathy: a randomized, double-blind study. Eur J Pain. 2008;12(2):203-13.

29. Food Drug Administration Centre for Drugs Evaluation Research. Highlights of Prescribing Information (LYRICA) Pregabalin. FDA Maryland; 2012.

30. MacDermott AB. Glutamate and GABA: a painful combination. Neuron. 2001;32(3):376-8.

31. Bardoni R, Takazawa T, Tong CK, Choudhury P, Scherrer G, Macdermott AB. Pre- and postsynaptic inhibitory control in the spinal cord dorsal horn. Ann N Y Acad Sci. 2013;1279:90-6.

32. Zeilhofer HU, Wildner H, Yevenes GE. Fast synaptic inhibition in spinal sensory processing and pain control. Physiol Rev. 2012;92(1):193-235.

33. Coull JA, Boudreau D, Bachand K, Prescott SA, Nault F, Sik A, et al. Trans-synaptic shift in anion gradient in spinal lamina I neurons as a mechanism of neuropathic pain. Nature. 2003;424(6951):938-42.

34. Li Z, Taylor CP, Weber M, Piechan J, Prior F, Bian F, et al. Pregabalin is a potent and selective ligand for alpha(2)delta-1 and alpha(2)delta-2 calcium channel subunits. Eur J Pharmacol. 2011;667(1-3):80-90.

35. Patel R, Bauer CS, Nieto-Rostro M, Margas W, Ferron $\mathrm{L}$, Chaggar $\mathrm{K}$, et al. alpha2delta-1 gene deletion affects somatosensory neuron function and delays mechanical hypersensitivity in response to peripheral nerve damage. $J$ Neurosci. 2013;33(42):16412-26.

36. Field MJ, Cox PJ, Stott E, Melrose H, Offord J, Su TZ, et al. Identification of the alpha2-delta- 1 subunit of voltage-dependent calcium channels as a molecular target for pain mediating the analgesic actions of 
pregabalin. Proc Natl Acad Sci USA. 2006;103(46):17537-42.

37. Ben-Menachem E. Pregabalin pharmacology and its relevance to clinical practice. Epilepsia. 2004;45(Suppl 6):13-8.

38. Hong T, Han S, Lee J, Jeon S, Yim DS. Comparison of oral absorption models for pregabalin: usefulness of transit compartment model. Drug Des, Dev Ther. 2016;10:3995-4003.

39. Bockbrader HN, Radulovic LL, Posvar EL, Strand JC, Alvey CW, Busch JA, et al. Clinical pharmacokinetics of pregabalin in healthy volunteers. J Clin Pharmacol. 2010;50(8):941-50.

40. Bockbrader HN, Burger P, Knapp L, Corrigan BW. Population pharmacokinetics of pregabalin in healthy subjects and patients with chronic pain or partial seizures. Epilepsia. 2011;52(2):248-57.

41. Satoh J, Yagihashi S, Baba M, Suzuki M, Arakawa A, Yoshiyama T. Efficacy and safety evaluation of pregabalin treatment over 52 weeks in patients with diabetic neuropathic pain extended after a doubleblind placebo-controlled trial. J Diabetes Investig. 2011;2(6):457-63.

42. Satoh J, Yagihashi S, Baba M, Suzuki M, Arakawa A, Yoshiyama T, et al. Efficacy and safety of pregabalin for treating neuropathic pain associated with diabetic peripheral neuropathy: a 14 week, randomized, double-blind, placebo-controlled trial. Diabetic Med J Br Diabetic Assoc. 2011;28(1):109-16.

43. Zaccara G, Gangemi P, Perucca P, Specchio L. The adverse event profile of pregabalin: a systematic review and meta-analysis of randomized controlled trials. Epilepsia. 2011;52(4):826-36.

44. Parsons B, Emir B. Glycemic and serum lipid control in patients with painful diabetic peripheral neuropathy treated with pregabalin. J Diabetes Complicat. 2017;31(2):489-93.

45. Verma V, Singh N, Singh Jaggi A. Pregabalin in neuropathic pain: evidences and possible mechanisms. Curr Neuropharmacol. 2014;12(1):44-56.

46. Rosenstock J, Tuchman M, LaMoreaux L, Sharma U. Pregabalin for the treatment of painful diabetic peripheral neuropathy: a double-blind, placebocontrolled trial. Pain. 2004;110(3):628-38.

47. Lesser H, Sharma U, LaMoreaux L, Poole RM. Pregabalin relieves symptoms of painful diabetic neuropathy: a randomized controlled trial. Neurology. 2004;63(11):2104-10.
48. Richter RW, Portenoy R, Sharma U, Lamoreaux L, Bockbrader H, Knapp LE. Relief of painful diabetic peripheral neuropathy with pregabalin: a randomized, placebo-controlled trial. J Pain. 2005;6(4):253-60.

49. Freynhagen R, Strojek K, Griesing T, Whalen E, Balkenohl M. Efficacy of pregabalin in neuropathic pain evaluated in a 12-week, randomised, doubleblind, multicentre, placebo-controlled trial of flexible- and fixed-dose regimens. Pain. 2005;115(3):254-63.

50. Zhang SS, Wu Z, Zhang LC, Zhang Z, Chen RP, Huang $\mathrm{YH}$, et al. Efficacy and safety of pregabalin for treating painful diabetic peripheral neuropathy: a meta-analysis. Acta Anaesthesiol Scand. 2015;59(2):147-59.

51. Tassone DM, Boyce E, Guyer J, Nuzum D. Pregabalin: a novel gamma-aminobutyric acid analogue in the treatment of neuropathic pain, partial-onset seizures, and anxiety disorders. Clin Ther. 2007;29(1):26-48.

52. Gilron I, Wajsbrot D, Therrien F, Lemay J. Pregabalin for peripheral neuropathic pain: a multicenter, enriched enrollment randomized withdrawal placebo-controlled trial. Clin J Pain. 2011;27(3):185-93.

53. Serpell M, Latymer M, Almas M, Ortiz M, Parsons B, Prieto R. Neuropathic pain responds better to increased doses of pregabalin: an in-depth analysis of flexible-dose clinical trials. J Pain Res. 2017;10:1769-76.

54. Bansal D, Bhansali A, Hota D, Chakrabarti A, Dutta P. Amitriptyline vs. pregabalin in painful diabetic neuropathy: a randomized double blind clinical trial. Diabet Med. 2009;26(10):1019-26.

55. Boyle J, Eriksson ME, Gribble L, Gouni R, Johnsen S, Coppini DV, et al. Randomized, placebo-controlled comparison of amitriptyline, duloxetine, and pregabalin in patients with chronic diabetic peripheral neuropathic pain: impact on pain, polysomnographic sleep, daytime functioning, and quality of life. Diabetes Care. 2012;35(12):2451-8.

56. Quilici S, Chancellor J, Löthgren M, Simon D, Said $\mathrm{G}$, Le TK, et al. Meta-analysis of duloxetine vs pregabalin and gabapentin in the treatment of diabetic peripheral neuropathic pain. BMC Neurol. 2009;9(1):6.

57. Finnerup NB, Otto M, McQuay HJ, Jensen TS, Sindrup SH. Algorithm for neuropathic pain treatment: an evidence based proposal. Pain. 2005;118(3):289-305. 
58. de Salas-Cansado M, Pérez C, Saldaña MT, Navarro A, González-Gómez FJ, Ruiz L, et al. An economic evaluation of pregabalin versus usual care in the management of community-treated patients with refractory painful diabetic peripheral neuropathy in primary care settings. Primary Care Diabetes. 2012;6(4):303-12.

59. Rodríguez MJ, Díaz S, Vera-Llonch M, Dukes E, Rejas J. Cost-effectiveness analysis of pregabalin versus gabapentin in the management of neuropathic pain due to diabetic polyneuropathy or post-herpetic neuralgia. Curr Med Res Opin. 2007;23(10):2585-96.

60. Iqbal Z, Azmi S, Yadav R, Ferdousi M, Kumar M, Cuthbertson DJ, et al. Diabetic peripheral neuropathy: epidemiology, diagnosis, and pharmacotherapy. Clin Ther. 2018;40(6):828-49.

61. Tesfaye S, Wilhelm S, Lledo A, Schacht A, Tolle T, Bouhassira D, et al. Duloxetine and pregabalin: high-dose monotherapy or their combination? The "COMBO-DN study"-a multinational, randomized, double-blind, parallel-group study in patients with diabetic peripheral neuropathic pain. Pain. 2013;154(12):2616-25.

62. Bouhassira D, Wilhelm S, Schacht A, Perrot S, Kosek E, Cruccu G, et al. Neuropathic pain phenotyping as a predictor of treatment response in painful diabetic neuropathy: data from the randomized, doubleblind, COMBO-DN study. Pain ${ }^{\circledR}$. 2014;155(10):2171-9.

63. Rolim LC, Koga da Silva EM, De Sá JR, Dib SA. A systematic review of treatment of painful diabetic neuropathy by pain phenotype versus treatment based on medical comorbidities. Front Neurol. 2017;8:285.

64. Boyle J, Eriksson MEV, Gribble L, Gouni R, Johnsen $\mathrm{S}$, Coppini DV, et al. Randomized, placebo-controlled comparison of amitriptyline, duloxetine, and pregabalin in patients with chronic diabetic peripheral neuropathic pain. Impact on pain, polysomnographic sleep, daytime functioning, and quality of life. 2012.

65. Baron R, Mayoral V, Leijon G, Binder A, Steigerwald I, Serpell M. Efficacy and safety of combination therapy with 5\% lidocaine medicated plaster and pregabalin in post-herpetic neuralgia and diabetic polyneuropathy. Curr Med Res Opin. 2009;25(7):1677-87.

66. Zin CS, Nissen LM, O'Callaghan JP, Duffull SB, Smith MT, Moore BJ. A randomized, controlled trial of oxycodone versus placebo in patients with postherpetic neuralgia and painful diabetic neuropathy treated with pregabalin. J Pain. 2010;11(5):462-71.
67. Tarride JE, Gordon A, Vera-Llonch M, Dukes E, Rousseau C. Cost-effectiveness of pregabalin for the management of neuropathic pain associated with diabetic peripheral neuropathy and postherpetic neuralgia: a Canadian perspective. Clin Ther. 2006;28(11):1922-34.

68. Galer BS, Gianas A, Jensen MP. Painful diabetic polyneuropathy: epidemiology, pain description, and quality of life. Diabetes Res Clin Pract. 2000;47(2):123-8.

69. Benbow SJ, Wallymahmed ME, Macfarlane IA. Diabetic peripheral neuropathy and quality of life. QJM Mon J Assoc Phys. 1998;91(11):733-7.

70. Atkinson JH, Ancoli-Israel S, Slater MA, Garfin SR, Dillin JC. Subjective sleep disturbance in chronic back pain. Clin J Pain. 1988;4(4):225-32.

71. Morin CM, Gibson D, Wade J. Self-reported sleep and mood disturbance in chronic pain patients. Clin J Pain. 1998;14(4):311-4.

72. Pilowsky I, Crettenden I, Townley M. Sleep disturbance in pain clinic patients. Pain. 1985;23(1):27-33.

73. Banks SM, Kerns RD. Explaining high rates of depression in chronic pain: a diathesis-stress framework. Psychol Bull. 1996;119(1):95-110.

74. Dworkin RH, Gitlin MJ. Clinical aspects of depression in chronic pain patients. Clin J Pain. 1991;7(2):79-94.

75. Fishbain DA, Cutler R, Rosomoff HL, Rosomoff RS. Chronic pain-associated depression: antecedent or consequence of chronic pain? A review. Clin J Pain. 1997;13(2):116-37.

76. Sullivan MJL, Reesor K, Mikail S, Fisher R. The treatment of depression in chronic low back pain: review and recommendations. Pain. 1992;50(1):5-13.

77. McWilliams LA, Cox BJ, Enns MW. Mood and anxiety disorders associated with chronic pain: an examination in a nationally representative sample. Pain. 2003;106(1-2):127-33.

78. Greenblatt HK, Greenblatt DJ. Gabapentin and pregabalin for the treatment of anxiety disorders. Clin Pharmacol Drug Dev. 2018;7(3):228-32.

79. Ogawa S, Arakawa A, Hayakawa K, Yoshiyama T. Pregabalin for neuropathic pain: why benefits could be expected for multiple pain conditions. Clin Drug Investig. 2016;36(11):877-88.

80. Ogawa S, Suzuki M, Arakawa A, Araki S, Yoshiyama T. Evaluation of the efficacy and safety of pregabalin 
in the treatment of postherpetic neuralgia: a randomized, double-blind, multicenter, placebo-controlled study. J Jpn Soc Pain Clin. 2010;17:141-52.

81. Cardenas DD, Nieshoff EC, Suda K, Goto S, Sanin L, Kaneko T, et al. A randomized trial of pregabalin in patients with neuropathic pain due to spinal cord injury. Neurology. 2013;80(6):533-9.

82. Baron R, Brunnmuller U, Brasser M, May M, Binder A. Efficacy and safety of pregabalin in patients with diabetic peripheral neuropathy or postherpetic neuralgia: open-label, non-comparative, flexible-dose study. Eur J Pain (London, England). 2008;12(7):850-8.

83. Hwang S, van Nooten F, Wells T, Ryan A, Crawford B, Evans C, et al. Neuropathic pain: a patient-centred approach to measuring outcomes. Health expectations: an international journal of public participation in health care and health policy. 2018.

84. Zelman DC, Brandenburg NA, Gore M. Sleep impairment in patients with painful diabetic peripheral neuropathy. Clin J Pain. 2006;22(8):681-5.

85. Sivertsen B, Lallukka T, Petrie KJ, Steingrimsdottir OA, Stubhaug A, Nielsen CS. Sleep and pain sensitivity in adults. Pain. 2015;156(8):1433-9.

86. Hagenauer MH, Crodelle JA, Piltz SH, Toporikova N, Ferguson P, Booth V. The Modulation of pain by circadian and sleep-dependent processes: a review of the experimental evidence. bioRxiv. 2017.

87. Gilron I, Bailey JM, Tu D, Holden RR, Weaver DF, Houlden RL. Morphine, gabapentin, or their combination for neuropathic. Pain. 2005;352(13):1324-34.

88. Gilron I, Bailey JM, Tu D, Holden RR, Jackson AC, Houlden RL. Nortriptyline and gabapentin, alone and in combination for neuropathic pain: a doubleblind, randomised controlled crossover trial. Lancet. 2009;374(9697):1252-61.

89. Gilron I, Bailey JM, Vandenkerkhof EG. Chronobiological characteristics of neuropathic pain: clinical predictors of diurnal pain rhythmicity. Clin J Pain. 2013;29(9):755-9.

90. Horner RL, Peever JH. Brain circuitry controlling sleep and wakefulness. Continuum (Minneap Minn). 2017;23(4, Sleep Neurology):955-72.

91. Taylor BK, Westlund KN. The noradrenergic locus coeruleus as a chronic pain generator. J Neurosci Res. 2017;95(6):1336-46.

92. Brightwell JJ, Taylor BK. Noradrenergic neurons in the locus coeruleus contribute to neuropathic pain. Neuroscience. 2009;160(1):174-85.
93. Garcia-Recio S, Gascón P. Biological and pharmacological aspects of the NK1-receptor. Biomed Res Int. 2015;2015:495704.

94. Muto Y, Sakai A, Sakamoto A, Suzuki H. Activation of $\mathrm{NK}(1)$ receptors in the locus coeruleus induces analgesia through noradrenergic-mediated descending inhibition in a rat model of neuropathic pain. Br J Pharmacol. 2012;166(3):1047-57.

95. Hayashida K, Obata H, Nakajima K, Eisenach JC. Gabapentin acts within the locus coeruleus to alleviate neuropathic pain. Anesthesiology. 2008;109(6):1077-84.

96. Lampl C, Schweiger C, Haider B, Lechner A. Pregabalin as mono- or add-on therapy for patients with refractory chronic neuropathic pain: a post-marketing prescription-event monitoring study. J Neurol. 2010;257(8):1265-73.

97. Ferini-Strambi L. Neuropathic pain and sleep: a review. Pain Ther. 2017;6(Suppl 1):19-23.

98. Vinik A, Emir B, Parsons B, Cheung R. Prediction of pregabalin-mediated pain response by severity of sleep disturbance in patients with painful diabetic neuropathy and post-herpetic neuralgia. Pain Med. 2014;15(4):661-70.

99. Roth T, van Seventer R, Murphy TK. The effect of pregabalin on pain-related sleep interference in diabetic peripheral neuropathy or postherpetic neuralgia: a review of nine clinical trials. Curr Med Res Opin. 2010;26(10):2411-9.

100. Coppini D. New NICE guidelines on diabetic foot disease prevention and management. Pract Diabetes. $2015 ; 32(8): 283$.

101. Richardson C, Glenn S, Nurmikko T, Horgan M. Incidence of phantom phenomena including phantom limb pain 6 months after major lower limb amputation in patients with peripheral vascular disease. Clin J Pain. 2006;22(4):353-8.

102. Mortimer CM, Steedman WM, McMillan IR, Martin DJ, Ravey J. Patient information on phantom limb pain: a focus group study of patient experiences, perceptions and opinions. Health Educ Res. 2002;17(3):291-304.

103. van der Schans CP, Geertzen JH, Schoppen T, Dijkstra PU. Phantom pain and health-related quality of life in lower limb amputees. J Pain Symptom Manage. 2002;24(4):429-36.

104. Vanross ER, Johnson S, Abbott CA. Effects of early mobilization on unhealed dysvascular transtibial amputation stumps: a clinical trial. Arch Phys Med Rehabil. 2009;90(4):610-7. 
105. McCormick Z, Chang-Chien G, Marshall B, Huang M, Harden RN. Phantom limb pain: a systematic neuroanatomical-based review of pharmacologic treatment. Pain Medicine. 2014;15(2):292-305.

106. Chen J, Li L, Chen SR, Chen H, Xie JD, Sirrieh RE, et al. The alpha2delta-1-NMDA receptor complex is critically involved in neuropathic pain development and gabapentin therapeutic actions. Cell Rep. 2018;22(9):2307-21.

107. Bauer CS, Nieto-Rostro M, Rahman W, Tran-VanMinh A, Ferron L, Douglas L, et al. The increased trafficking of the calcium channel subunit alpha2delta-1 to presynaptic terminals in neuropathic pain is inhibited by the alpha2delta ligand pregabalin. J Neurosci. 2009;29(13):4076-88.

108. Daiichi-Sankyo. Mirogabalin 2018. https://www. daiichisankyo.com/media_investors/media_relatio ns/press_releases/detail/006800.html.

109. Vinik A, Rosenstock J, Sharma U, Feins K, Hsu C, Merante D. Efficacy and safety of mirogabalin (DS5565) for the treatment of diabetic peripheral neuropathic pain: a randomized, double-blind, placeboand active comparator-controlled, adaptive proofof-concept phase 2 study. Diabetes Care. 2014;37(12):3253-61.

110. Fischer TZ, Waxman SG. Familial pain syndromes from mutations of the NaV1.7 sodium channel. Ann N Y Acad Sci. 2010;1184:196-207.

111. A Clinical Trial To Evaluate PF-05089771 on its own and as an Add-on Therapy to Pregabalin (Lyrica) for the Treatment of Pain due to Diabetic Peripheral Neuropathy (DPN). 2014 [cited 2018 June 28]. http://clinicaltrials.gov/ct2/show/NCT02215252? term $=$ PF-05089771\&rank=8.

112. Convergence Pharmaceuticals. Convergence Pharmaceuticals' Novel Nav1.7 Selective Sodium Channel Blocker CNV1014802 Demonstrates Proof of Concept in Second Neuropathic Pain Study in Lumbosacral Radiculopathy 2014 [cited 2018 June 28]. http://www.convergencepharma.com/userfiles/ file/140923_LSR_FINAL.pdf.

113. Winters CS, Yang M, Chen H. Pharmaceutical compositions of spiro-oxindole compound for topical administration and their use as therapeutic agents. PCT Int Apple WO2011106729. 2011.

114. Sumitomo Dainippon Pharma Co Ltd. Profile of Major Products under Development. http://www. ds-pharma.com/rd/clinical/pipeline_profile.html.

115. Kwong K, Webb EF, Hunsberger GE, et al. Pharmacological characterisation of GSK2339345, a novel voltage-gated sodium channel blocker for the symptomatic relief of cough. Am J Resp Grit Care Med. 2013;187:A4936.

116. ClinicalTrials.gov; U.S. National Institutes of Health: GS-6615 2015. https://clinicaltrials.gov/ct2/ results?term=GS6615\&Search=Search.

117. Specialist Pharmacy Service NHS. Mirogabalin 2017. https://www.sps.nhs.uk/medicines/mirogabalin/.

118. Kato M, Tajima N, Shimizu T, Sugihara M, Furihata $\mathrm{K}$, Harada $\mathrm{K}$, et al. Pharmacokinetics and safety of a single oral dose of mirogabalin in Japanese subjects with varying degrees of renal impairment. J Clin Pharmacol. 2018;58(1):57-63.

119. Mu Y, Liu X, Li Q, Chen K, Liu Y, Lv X, et al. Efficacy and safety of pregabalin for painful diabetic peripheral neuropathy in a population of Chinese patients: a randomized placebo-controlled trial. J Diabetes. 2018;10(3):256-65.

120. Huffman C, Stacey BR, Tuchman M, Burbridge C, Li C, Parsons B, et al. Efficacy and safety of pregabalin in the treatment of patients with painful diabetic peripheral neuropathy and pain on walking. Clin J Pain. 2015;31(11):946-58.

121. Raskin P, Huffman C, Yurkewicz L, Pauer L, Scavone JM, Yang R, et al. Pregabalin in patients with painful diabetic peripheral neuropathy using an NSAID for other pain conditions: a double-blind crossover study. Clin J Pain. 2016;32(3):203-10.

122. Raskin P, Huffman C, Toth C, Asmus MJ, Messig M, Sanchez RJ, et al. Pregabalin in patients with inadequately treated painful diabetic peripheral neuropathy: a randomized withdrawal trial. Clin J Pain. 2014;30(5):379-90.

123. Satoh J, Yagihashi S, Baba M, Suzuki M, Arakawa A, Yoshiyama T, et al. Efficacy and safety of pregabalin for treating neuropathic pain associated with diabetic peripheral neuropathy: a 14 week, randomized, double-blind, placebo-controlled trial. Diabet Med. 2010;28(1):109-16.

124. Guan Y, Ding X, Cheng Y, Fan D, Tan L, Wang Y, et al. Efficacy of pregabalin for peripheral neuropathic pain: results of an 8-week, flexible-dose, double-blind, placebo-controlled study conducted in China. Clin Ther. 2011;33(2):159-66.

125. Parsons B, Li C. The efficacy of pregabalin in patients with moderate and severe pain due to diabetic peripheral neuropathy. Curr Med Res Opin. 2016;32(5):929-37.

126. Freeman R, Emir B, Parsons B. Predictors of placebo response in peripheral neuropathic pain: insights from pregabalin clinical trials. J Pain Res. 2015;8:257-68. 
127. Freeman R, Durso-Decruz E, Emir B. Efficacy, safety, and tolerability of pregabalin treatment for painful diabetic peripheral neuropathy: findings from seven randomized, controlled trials across a range of doses. Diabetes Care. 2008;31(7): 1448-54.

128. Freynhagen R, Serpell M, Emir B, Whalen E, Parsons B, Clair A, et al. A comprehensive drug safety evaluation of pregabalin in peripheral neuropathic pain. Pain Pract. 2015;15(1):47-57.

129. Semel D, Murphy TK, Zlateva G, Cheung R, Emir B. Evaluation of the safety and efficacy of pregabalin in older patients with neuropathic pain: results from a pooled analysis of 11 clinical studies. BMC Family Practice. 2010;11:85.
130. Moon DE, Lee DI, Lee SC, Song SO, Yoon DM, Yoon $\mathrm{MH}$, et al. Efficacy and tolerability of pregabalin using a flexible, optimized dose schedule in Korean patients with peripheral neuropathic pain: a 10 -week, randomized, double-blind, placebo-controlled, multicenter study. Clin Ther. 2010;32(14):2370-85.

131. Arezzo JC, Rosenstock J, LaMoreaux L, Pauer L. Efficacy and safety of pregabalin $600 \mathrm{mg} / \mathrm{d}$ for treating painful diabetic peripheral neuropathy: a double-blind placebo-controlled trial. BMC Neurol. 2008;8:33.

132. Tölle T, Freynhagen R, Versavel M, Trostmann U, Young JP. Pregabalin for relief of neuropathic pain associated with diabetic neuropathy: a randomized, double-blind study. Eur J Pain. 2012;12(2):203-13. 